\title{
Integración Asistencial de Dos Organizaciones en Cataluña, España
}

\author{
Integral health provision by two Catalonian health \\ providing entities (Spain)
}

Diana Henao-Martínez ${ }^{1}$, María L. Vázquez-Navarrete ${ }^{1}$ Ingrid Vargas-Lorenzo ${ }^{1}$, Jordi Coderch-Lassaletta ${ }^{2}$ y Josep R. Llopart-López ${ }^{3}$

1 Servei d'Estudis i Prospectives en Polítiques de Salut del Consorci Hospitalari de Catalunya. Barcelona, España. dhenao@chc.es, mlvazquez@chc.es, ivargas@chc.es

2 Serveis de Salut Integrats Baix Empordà. Palamós, España. jcoderch@hosppal.es

3 Badalona Serveis Assistencials. Badalona, España. jrllopart@bsa.gs

Recibido 26 Julio 2007/Enviado para Modificación 7 Diciembre 2007/Aceptado 9 Enero 2008

\section{RESUMEN}

Objetivo El objetivo es analizar la experiencia de dos redes integradas de servicios de salud (RISS ) en Cataluña para identificar elementos de mejora.

Métodos Estudio de casos, mediante análisis de documentos y entrevistas individuales semi-estructuradas. Se seleccionaron dos casos -RISS- y para cada caso, documentos e informantes que proporcionaran información sobre las dimensiones de análisis. Se realizó análisis de contenido con generación mixta de categorías y segmentación por casos y temas. Trabajo de campo en 2004.

Resultados Ambas son organizaciones sanitarias integradas verticalmente hacia atrás, con producción interna total de servicios e integración virtual en la propiedad. Mientras la financiación de BSA es por línea de servicio, SSIBE participa en la prueba piloto de financiación capitativa. Ambas disponen de un gobierno múltiple estrechamente coordinado y han definido estrategias globales orientadas a la coordinación y la eficiencia, con diferencias en cuanto a tiempo de desarrollo. Mientras BSA poseía una estructura divisional, la de SSIBE es funcional por ámbitos transversales. La coordinación asistencial se basa en la normalización de procesos y de habilidades, con escasos mecanismos de adaptación mutua y disparidad en el número de instrumentos implementados.

Conclusiones Ambas organizaciones presentan elementos favorables y desfavorables a la coordinación asistencial, que requerirían cambios en los ámbitos interno y externo, que conduzcan hacia la eficiencia y la continuidad asistencial.

Palabras Clave: Servicios de salud, organización, estudio de caso. (fuente: DeCS, BIREME)

\section{ABSTRACT}

Objective Health policies aimed at promoting collaboration amongst providers have led to different initiatives, amongst them integrated healthcare delivery systems (IDS); these have been analysed mainly in the USA but hardly so in Colombia or Spain. This 
article thus analyses the experience of two IDS in Catalonia for identifying elements for improvement.

Methods This was a case-study carried out via individual semi-structured interviews and analysing documents. Two IDS were selected; a sample of documents and reports providing information on analysis variables were selected for each case. Content was analysed via mixed categories and segmentation by cases and topics. Results Both IDS are health-care providing organisations presenting backward vertical integration, having total internal service production and virtual integration of ownership. BSA is funded by providing services whilst SSIBE relies on shareholding via capitation pilot test. Both have closely coordinated multiple managing bodies and have defined overall strategies orientated towards coordination and efficiency; they differ regarding implementation time. BSA has a divisional structure and SSIBE a functional one, organised by transversal areas. Clinical coordination is based on standardising processes and abilities, having few mechanisms for mutual adaptation and disparity in the number of instruments implemented.

Conclusions Both organisations presented enabling and hindering factors for clinical coordination which would need changes in internal and external components in order to improve overall efficiency and health care continuity.

Key Words: Integrated delivery system, health care, case study (source: MeSH, $N L M)$.

$\mathrm{D}$ esde los años 90, países de todo el mundo han introducido reformas en la financiación y organización de la provisión de servicios de salud, basadas en la introducción de mecanismos de mercado. En América Latina, las reformas surgieron como un intento de responder a problemas crónicos de ineficiencia e inequidad (1). En los últimos años, sin embargo, los discursos dominantes en las políticas de salud, pasaron de promocionar la competencia a la búsqueda de la colaboración entre los proveedores de salud, para mejorar la continuidad asistencial y la eficiencia global del sistema (2). Con este objetivo, tanto países desarrollados, como en desarrollo realizaron experiencias diversas (3), que incluyen las redes integradas de servicios de salud (RISS) (4) u organizaciones sanitarias integradas (OSI)(5). Una RISS es un conjunto de organizaciones que ofrecen una atención coordinada a través del continuo de servicios de salud a una población determinada y es responsable de los resultados económicos y en salud (6). Se distinguen dos tipos: a) de afiliación geográfica y b) de afiliación voluntaria (7). Las primeras se producen cuando hay una devolución de la gestión de los servicios de salud del gobierno central a un nivel inferior de gobierno -autoridad regional de salud, municipio, etc. Se caracteriza por contar con una población definida geográficamente y, generalmente, formar parte de un sistema nacional de salud. El segundo tipo se crea en países que han establecido un mercado para el aseguramiento de la 
salud donde la población tiene que comprar sus planes de salud (8,9). En estos países, las fuerzas del mercado y las reformas han conducido a una progresiva integración de aseguradoras y proveedores en una única entidad (10).

Las RISS han aparecido, sobre todo, en países con sistemas de salud privados muy fragmentados, como EEUU, y en países con sistemas de seguros sociales en los que aseguradoras y proveedores compiten por afiliados (10). También se han promovido en países con sistemas nacionales de salud, con diversificación en la titularidad de los proveedores, como España o Reino Unido $(5,11)$. En Latinoamérica han sido introducidas en Colombia, Chile, Argentina, Perú, Brasil y el Salvador (12).

En Colombia, las Leyes 60 y 100 de 1993 crearon el marco para la descentralización de la gestión del aseguramiento y la introducción de la competencia en el sistema de salud. El núcleo de la reforma era el establecimiento de un intermediario -las aseguradoras- con funciones de afiliación, recaudación y organización de la provisión de servicios: las Entidades Promotoras de Salud (EPS) para el Régimen Contributivo y las Administradoras del Régimen Subsidiado (ARS). Garantizan la provisión, directamente o mediante otros proveedores, de un paquete de prestaciones en salud, diferente para cada régimen (4).

El sistema nacional de salud en España se financia por impuestos generales, es gratuito en el punto de dispensación de la atención, con cobertura universal de un amplio paquete de prestaciones, propiedad esencialmente pública de la provisión, cierta autonomía de gestión para los proveedores de salud y modelo de atención basado en la atención primaria (13). Cataluña, una de las comunidades autónomas, introdujo la separación de las funciones de financiación y provisión de servicios de salud (14). El Servei Català de la Salut (CatSalut), entidad pública dependiente del Departament de Salut (máxima autoridad del sistema), compra los servicios a proveedores concertados de titularidad diversa -empresas públicas, consorcios, fundaciones sin ánimo de lucro, etc.-, que conforman 70 \% de la atención hospitalaria y $20 \%$ de la atención primaria de la red pública (15). El resto está provisto por el Institut Català de la Salut (ICS), propiedad de la Generalitat. La provisión se organiza por demarcaciones territoriales. La relación entre el financiador público y los proveedores de salud se establece mediante los contratos de gestión; sin intermediarios. El CatSalut aplica un sistema de compra por líneas de servicios -atención primaria, atención especializada y sociosanitaria- y, desde el 2001, puso en marcha un proyecto piloto para la compra de servicios en base poblacional (15). 
Estados Unidos ha sido pionero en la investigación de las RISS (16-18). En cambio, las RISS surgidas en España, en un sistema nacional de salud, con características particulares, han sido escasamente analizadas $(5,19)$. El objetivo del artículo, que presenta resultados parciales de una investigación más extensa $(20,21)$, es analizar la experiencia de dos RISS en Cataluña para identificar elementos de mejora.

\section{SUJETOS Y MÉTODOS}

Diseño del estudio. Estudio de casos cualitativo, exploratorio (22), basado en la aplicación de la "Guía para el estudio de OSI de base territorial" (23). El trabajo de campo se realizó entre abril y septiembre de 2004.

Muestra. Se diseñó una muestra en dos etapas, en la primera se eligieron los casos, las RISS, según los criterios: ser proveedor integrado verticalmente; más de cinco años de gestión conjunta; población de referencia definida y un hospital comarcal en la red. Los casos fueron: Badalona Serveis Assistencials (BSA) y Serveis de Salut Integrats Baix Empordà (SSIBE). En la segunda etapa, para cada RISS se diseñó una muestra teórica (22) de documentos que pudieran proporcionar información sobre los elementos que influyen en la coordinación asistencial. Se analizaron 80 documentos: estatutos, memorias, contratos, actas, convenios -30-; planes, modelos organizativos, guías de práctica clínica -32-, y otros -18. Para completar la información, se seleccionaron distintos informantes: directivos de la RISS -6-, directivos de unidades operativas -2-, técnicos de apoyo y profesionales -4. La selección de los informantes se hizo mediante muestreo nominado (22). El tamaño final se alcanzó por saturación de la información.

Técnicas de recogida de la información. Análisis de documentos: con base en la guía (23), se elaboró el instrumento de análisis de documentos. Las dimensiones analizadas correspondían a la evolución histórica, elementos del entorno e internos que influyen sobre la continuidad asistencia. Entrevistas individuales. Se realizaron entrevistas individuales semi-estructuradas, con guía de entrevista, para completar y profundizar la información obtenida del análisis de documentos sobre las dimensiones consignadas en el párrafo anterior. Las entrevistas, de 90 minutos aproximadamente, se registraron manualmente.

Análisis y calidad de los datos. Se realizó análisis de contenido de documentos y entrevistas, con generación mixta de categorías a partir de los instrumentos y las emergidas de los datos. Se segmentaron los datos por caso y dimensiones 
de análisis. Las dimensiones analizadas fueron: del entorno -financiación, población y presencia de otros proveedores en el territorio-; del ámbito interno -gobierno, dimensión estratégica, estructura organizativa, asignación interna de recursos e incentivos y coordinación asistencial (24).

La calidad de los datos se aseguró con la triangulación a través de comparaciones entre técnicas y analistas con buen conocimiento del contexto. La procedencia de los datos de identifica mediante referencia. El proyecto fue aprobado por el comité de ética del Consorci Hospitalari de Catalunya.

\section{RESULTADOS}

\section{Badalona Serveis Assistencials}

Descripción y tipo de RISS. BSA es una organización sanitaria conformada por tres entidades: dos sociedades anónimas y una sociedad limitada. Brinda atención del continuo asistencial -atención primaria, especializada de agudos y sociosanitaria- al área de referencia hospitalaria, con una población de 108869 habitantes (25), un 26 \% de la región sanitaria Barcelonès Nord, al 100 \% de la cual proporciona atención sociosanitaria (26).

La constitución de las sociedad limitada para gestionar la atención primaria, en 1994 (Escriptura de constitució de societat otorgada per "Badalona Gestió Assistencial, S.L.". 1993), representó el inicio de la integración de servicios de los diferentes niveles asistenciales. Las tres entidades agrupadas bajo la marca BSA eran gestionadas por una empresa externa, con gerente única (25), pero mantenían personería jurídica propia, siendo una de las sociedades propiedad del ayuntamiento de Badalona y propietaria de las otras dos. En el 2005 se fusionaron en una única empresa municipal (entrevista. directivo RISS). El análisis que se presenta es previo a dicha fusión.

Por los niveles asistenciales que integra, BSA es una organización con integración vertical y hacia atrás, pues la integración partió del hospital hacia la atención primaria; con integración total por producir internamente todos los servicios que provee y virtual, por mantener la independencia en la propiedad de las entidades.

Elementos del entorno. En la región sanitaria del Barcelonès Nord, existen otros proveedores de salud: el ICS, provee atención primaria y atención especializada, con un hospital que actúa como hospital general básico para el 
33 \% de la población y además es hospital de referencia para la región(27). En salud mental de adultos, un instituto municipal atiende la población de Badalona (28). BSA no había desarrollado ningún mecanismo específico de coordinación asistencial con estas entidades. El CatSalut establecía contratos con cada entidad de BSA por líneas de servicios.

Elementos internos. Cada entidad posee su órgano de gobierno (Estatuts de la societat. BSA, S.L. 1993), dos constituidos por políticos locales, y uno por la gerente. Se coordinan mediante la presencia de algunos miembros en ambos y la participación de la gerente, con voz pero sin voto. Sus funciones, definidas en los estatutos, son la aprobación de presupuestos y gastos, de la plantilla de personal y del plan anual de las entidades, entre otras.

La dimensión estratégica de BSA se expresa en el plan estratégico que contiene la misión, las estrategias y los objetivos globales para cuatro años. Algunas de las estrategias y objetivos que se orientan hacia la coordinación y la eficiencia (Tabla 1) aún se encuentran en proceso de implantación. Los objetivos de las unidades operativas se derivan de los objetivos globales de la organización y se suman a los trazados por el comprador de servicios.

La estructura organizativa es divisional; cada división agrupa las actividades correspondientes a un nivel asistencial. Las funciones de apoyo, asistenciales y no asistenciales, están integradas para toda la red. Se coordinan mediante un órgano de gestión único formado por la gerente y las direcciones asistenciales y de apoyo (entrevista directivo 2004).

Tabla 1. Estrategias globales para mejorar la coordinación y la eficiencia

\begin{tabular}{|c|c|c|}
\hline Estratogias & BSA & SSIBE \\
\hline \multirow[t]{6}{*}{ Coordinación } & Alianzas con otros proweedores de salud & $\begin{array}{l}\text { Alianzas con otros provendores } \\
\text { de salud }\end{array}$ \\
\hline & Plan estrategica para toda la RISS & $\begin{array}{l}\text { Planificación estrategica para } \\
\text { toda la RISS }\end{array}$ \\
\hline & $\begin{array}{l}\text { Desarrollo de un sistema de información } \\
\text { con historia clinica informatizada } \\
\text { compartida }\end{array}$ & $\begin{array}{l}\text { Desarrollo de un sistema de } \\
\text { información con historia clinica } \\
\text { informatizada ûnica }\end{array}$ \\
\hline & Estrategia de gestićn compartida & Estrategia de gestión compartida \\
\hline & Mecanismos de coordinacion asistencial & $\begin{array}{l}\text { Mecanismos de cocrdinación } \\
\text { asistencial }\end{array}$ \\
\hline & Incentives alineados con pbjetives & $\begin{array}{l}\text { Incentivas alineados con } \\
\text { objebivos }\end{array}$ \\
\hline Eficlencia & Gulas de práctica clinica compartidas & $\begin{array}{l}\text { Guias de practica dínica } \\
\text { compartides }\end{array}$ \\
\hline $\begin{array}{l}\text { Coardinación y } \\
\text { eficiancia }\end{array}$ & $\begin{array}{l}\text { Monitorización de la red con cuadro de } \\
\text { mando integral }\end{array}$ & $\begin{array}{l}\text { Aumentar la cepacided resclutiva } \\
\text { de atención primaria }\end{array}$ \\
\hline
\end{tabular}

BSA: Badalona Serveis Assistencials; SSIBE: Serveis de Salut Integrats Baix Empordà; RISS: Red integrada de salud 


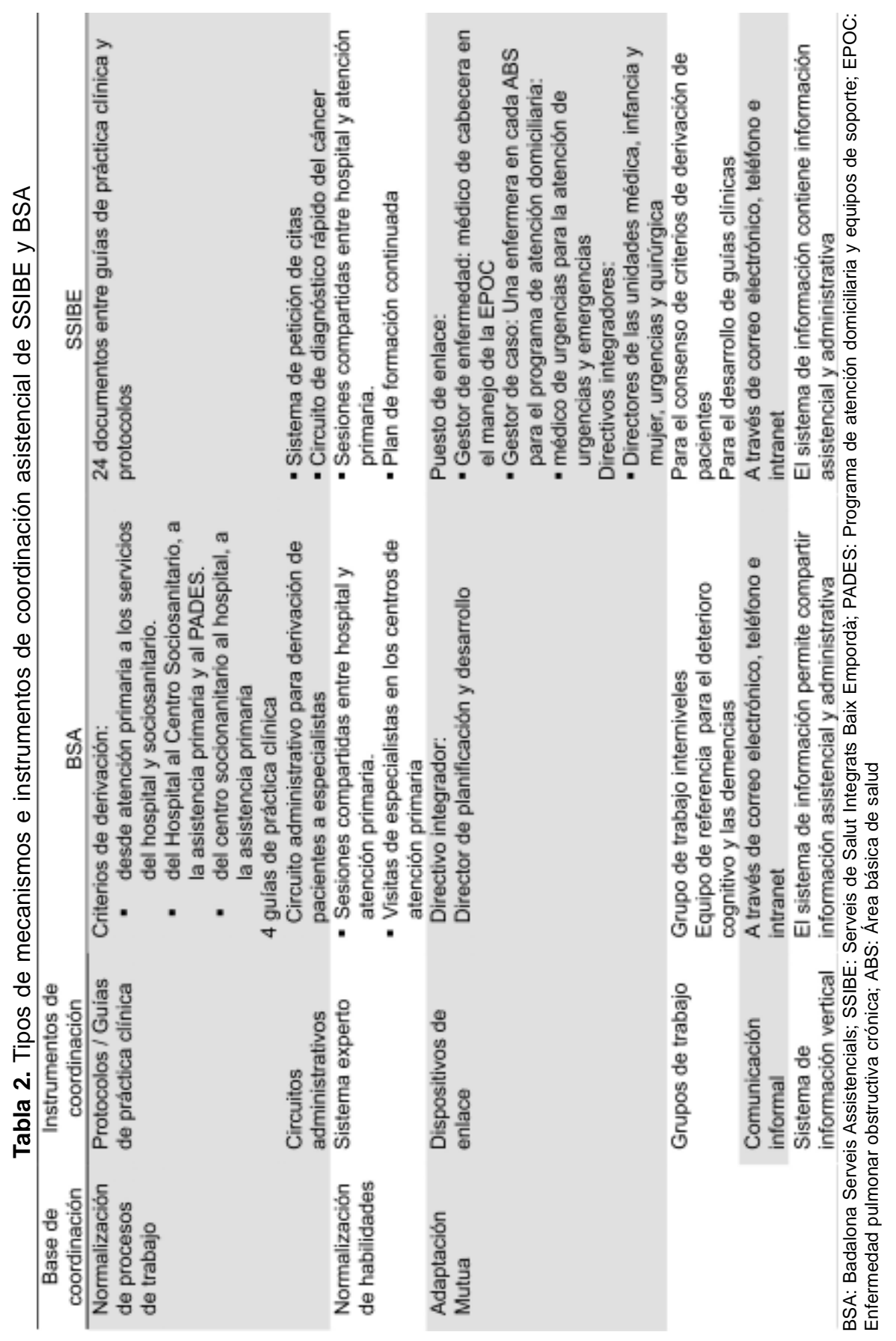


Para asignar los incentivos, la gerencia pacta contratos de gestión con los servicios del hospital y del sociosanitario que especifican, entre otros, objetivos relacionados con la coordinación entre niveles y la eficiencia y las condiciones para la asignación de incentivos según el logro de los objetivos (Contracte de gestió 2004). Con atención primaria se pacta un porcentaje variable del sueldo que dependerá del resultado de indicadores individuales, basados en los objetivos operativos anuales (entrevista directivo 2004)

Coordinación asistencial. En BSA, como en el resto del sistema de salud, la atención primaria es la puerta de entrada a los servicios y, según la complejidad, soluciona el problema o lo deriva a la atención especializada, ofrecida en un 50 $\%$ en centros de atención primaria y un 50 \% en centros hospitalarios. Para la coordinación entre niveles utiliza distintos mecanismos correspondientes a tres grandes grupos, normalización de habilidades, de procesos y adaptación mutua (Tabla 2). La atención primaria y el hospital de agudos se coordinan, principalmente, a través de sesiones compartidas de los especialistas del hospital con los profesionales de primaria; atención primaria y sociosanitaria, a través del programa de atención domiciliaria. El equipo de soporte sociosanitario en geriatría, facilita la conexión entre el hospital de agudos y la atención domiciliaria. Actualmente se está desarrollando un nuevo programa, Servei d'Atenció Integral a Domicili, que actuará como dispositivo de enlace para coordinar todos los niveles asistenciales en la atención domiciliaria (plan estratégico 2004-2007). En cuanto al sistema de información, BSA utiliza dos historias clínicas compartidas entre los niveles asistenciales. El sistema contiene los aplicativos específicos para cada servicio, la historia clínica informatizada e información de la organización, a la cual los profesionales asistenciales y administrativos acceden a través de la red interna, en distintos grados según el nivel asistencial. Se encuentra en evolución.

Serveis de Salut Integrats Baix Empordà (SSIBE)

Descripción y tipo de RISS. La red se inicia en 1994, con la creación del Consorci Asistencial del Baix Empordà (CABE) para gestionar la atención primaria. SSIBE está conformada por tres entidades: una fundación privada, un consorcio público (constituido por el consejo comarcal del Baix Empordà y la Fundación) y una asociación de interés económico, a través de la cual se relacionan. La organización brinda los servicios del continuo asistencial -atención primaria, de agudos y a la dependencia- al 72 \% de la población de referencia hospitalaria, abarcando todos los municipios de la comarca, excepto uno en atención primaria. 
Por el tipo de servicios que integra, SSIBE es una red de integración vertical hacia atrás ya que la integración se ha iniciado desde el hospital a la atención primaria; integración total por producir directamente todos los servicios que le son contratados y una red virtual por la forma de relación entre las entidades que la constituyen.

Elementos del entorno. En la comarca del Baix Empordà también proporcionan servicios otros proveedores: el ICS, la atención primaria a aproximadamente 30 \% de la población y el Instituto de Asistencia Sanitaria (IAS), la atención de salud mental y drogodependencias para toda la población, con quienes SSIBE comparte la historia clínica, sesiones clínicas conjuntas con la primaria e interconsultas (29). Con el ICS, participa conjuntamente en la prueba piloto de financiación per capita (30), donde se comprometen conjuntamente a proporcionar una cartera de servicios de salud para la población del territorio. No obstante, el CatSalut mantiene los objetivos y la facturación por líneas de servicios y entidades (entrevista directivo 2004).

Elementos internos. SSIBE posee tres órganos de gobierno independientes jurídicamente formados por autoridades políticas comarcales, municipales y autonómicas, el párroco y vecinos de Palamós. Se coordinan mediante la presencia de la mayoría de los miembros en los tres y del gerente, con voz pero sin voto, y reuniones conjuntas. Según las actas, los órganos de gobierno cumplen con sus funciones -aprobar cuentas y presupuestos y velar por la aplicación de los estatutos- y además discuten temas institucionales, asistenciales, relacionados con recursos humanos y laborales.

En su dimensión estratégica, SSIBE ha definido una misión, visión, estrategias y objetivos institucionales y anuales conjuntos (misión, visión, principios institucionales y estrategias, 2001), orientados a la consolidación de un sistema integrado de servicios sanitarios y sociales. Las estrategias incluyen algunas dirigidas a la coordinación y la eficiencia, que ya han sido implementadas (Tabla 1). Partiendo de los principios institucionales y los objetivos del comprador, se definen los objetivos anuales de la organización, mediante un sistema escalonado de los órganos de gestión a los profesionales de base (entrevista directivo 2004).

La estructura organizativa de SSIBE es funcional con integración de la dirección asistencial por ámbitos transversales, unidades asistenciales (médica, infancia-mujer, urgencias y quirúrgica), que agrupan todos los servicios relacionados con independencia del nivel asistencial. De su director dependen 
las direcciones de atención primaria y del sociosanitario y los jefes de servicio, (Pla funcional 2002). Las funciones de dirección se coordinan mediante dos órganos de gestión, el Consejo de Dirección y la Dirección Asistencial. Las funciones de apoyo se hallan centralizadas.

SSIBE aplica la dirección por objetivos, asignando incentivos económicos según su cumplimiento. Se aplican a todos los profesionales de atención primaria y sólo a directivos, jefes de servicio y mandos intermedios (entrevista directivo 2004).

Coordinación asistencial. El modelo organizativo asistencial se basa en el modelo del sistema nacional de salud en que se inserta, con la atención primaria como puerta de entrada y filtro del sistema y la atención especializada prestando servicios temporales de corta, media y larga estancia y procedimientos diagnósticos especializados. SSIBE utiliza mecanismos de coordinación asistencial entre niveles de diversos tipos -normalización de habilidades, de procesos y adaptación mutua (Tabla 2). Cuentan con dos programas específicos con características de dispositivos de enlace -la atención domiciliaria y de urgencias que coordinan la atención relacionada (entrevistas profesionales 2005). El sistema de información es propio y unifica la historia clínica para todos los niveles asistenciales. Permite el acceso a los datos clínicos, actividades pendientes, gestión de las prescripciones de farmacia y vistas del usuario (31), en diversos grados según el nivel asistencial. Se encuentra en evolución.

\section{DISCUSIÓN}

El estudio permite profundizar en las características de las RISS catalanas y su adaptación para la gestión conjunta de la provisión del continuo asistencial en un sistema nacional de salud. Las organizaciones analizadas presentan una tipología similar y, al mismo tiempo, elementos singulares. Por los niveles asistenciales que integran, son organizaciones con integración vertical hacia atrás por haber realizado la integración desde el hospital de agudos hacia la atención primaria. Integración que puede proporcionar los beneficios propios de economías de escala y que exige una estrecha coordinación entre los servicios y niveles asistenciales (32). En ambos casos los procesos de integración han sido liderados por los hospitales. Según la literatura (33), cuando las redes derivan de una organización hospitalaria suelen encontrar dificultades para romper con la cultura hospitalocentrista; se espera, no obstante, que sean capaces de adaptarse a un esquema que fortalezca el papel de la atención primaria, sin perder su experiencia en gestión. SSIBE, según resultados de otro 
trabajo, trató de apoyar la atención primaria desde el inicio con la descentralización de pruebas diagnósticas e implantación de servicios de urgencias en primaria y la promoción del rol consultor de los especialistas (19). Por mantener la personería jurídica de las entidades son redes virtuales. No obstante, la fusión en 2005, convirtió a BSA en una red con integración real, por ejercer control directo sobre todas las partes (34).

La asignación de fondos, por parte del comprador, por líneas de servicios con objetivos específicos y formas de pago diferentes es un elemento del entorno que no favorece la coordinación asistencial, ya que condiciona a los proveedores a ejecutar el presupuesto según los contratos, pudiendo ir contra los objetivos globales de la organización. Así, un aumento en la capacidad de resolución de la primaria, llevaría a una disminución de los ingresos económicos del hospital, que dependen de la actividad asistencial. En este sentido, un sistema de pago capitativo con un diseño adecuado, podría incentivar a los proveedores a buscar fórmulas que les permita alinear sus intereses para alcanzar los objetivos globales del sistema, reducir costos y fortalecer la calidad del servicio (35). Sólo SSIBE había establecido algunos mecanismos de coordinación conjuntos con los proveedores de la zona, y, por su participación en la prueba piloto de financiación per capita había desarrollado una alianza, aunque con muy limitadas implicaciones, según la evaluación de la prueba (36). La presencia limitada de mecanismos de coordinación con los otros proveedores del territorios podría condicionar un acceso desigual de la población a las atenciones oportunas y adecuadas y afectar la continuidad asistencial (37), a pesar de encontrarse inmersos en un sistema nacional de salud.

Pese a poseer múltiples órganos de gobierno, en ambas organizaciones operan como uno solo, lo que según la literatura (38) aporta diversas ventajas: claridad de objetivos, unidad de mando y compromiso con la totalidad del sistema que gobiernan. En su composición destaca la representación de las distintas entidades y de miembros con influencia política en el territorio, considerados atributos de buena gobernabilidad que deberían facilitar conocimiento de las necesidades de la población (39).

SSIBE intenta romper la separación entre niveles asistenciales integrando los diferentes niveles en una dirección única. En cambio, el tipo de estructura divisional por niveles asistenciales- de BSA mantiene el equilibrio de poder entre los niveles asistenciales pero con el riesgo de la descoordinación en la toma de decisiones, que la organización trata de contrarrestar con la figura del directivo integrador (40). La integración de las funciones de dirección y de 
apoyo, se considera facilitador de la coordinación de la atención (41), en la medida en que contribuye a la integración asistencial (42).

Ambas organizaciones utilizan diversos mecanismos de coordinación entre los distintos niveles asistenciales. SSIBE priorizó los instrumentos derivados de la normalización de procesos de trabajo y de adaptación mutua, mientras BSA promovió los primeros e introdujo limitadamente los últimos. En ambos casos ha habido un importante desarrollo del sistema de información, y se comparte la información del paciente entre niveles asistenciales, con grados diversos de implantación y agilidad. Según la opinión de directivos y profesionales, los diversos mecanismos de coordinación aun presentan limitaciones en su implementación y constituyen una de las principales barreras internas de la coordinación (43). Se debería profundizar también en la percepción de los usuarios de los servicios, para determinar si los cambios organizativos se traducen en una mejor continuidad asistencial.

Conclusiones y lecciones aprendidas

Las organizaciones analizadas presentan tanto elementos favorables como desfavorables para la coordinación asistencial. En su evolución, deberían fortalecer las estructuras que les permita aumentar la capacidad resolutiva de la atención primaria, implementar o aumentar las alianzas con los otros proveedores de salud del territorio y avanzar en el desarrollo de instrumentos que orienten las decisiones de los profesionales de diferentes niveles de manera coordinada, para mejorar la eficiencia y la continuidad asistencial.

Los sistemas de salud de Colombia y Cataluña presentan diferencias marcadas: segmentación en el aseguramiento y la provisión, con paquetes de beneficios diferenciados según capacidad adquisitiva y privatización de la gestión del aseguramiento en el modelo colombiano y un sistema nacional de salud de cobertura universal y organizado territorialmente, en el ámbito catalán. No obstante, ambos sistemas se asemejan en la fragmentación en la titularidad de la provisión de servicios y la tendencia a la formación de redes para mantener la continuidad en la atención.

Es importante destacar que la formación de redes integradas de servicios de salud no es un objetivo en sí mismo, sino una estrategia, entre otras posibles, para mejorar la continuidad asistencial y la eficiencia global. Podrían jugar un papel en el caso de sistemas que presentan segmentación de la provisión. No obstante, su contribución dependerá del grado en que el sistema garantice la 
financiación y acceso real de la población a la atención en salud. En un sistema nacional de salud, la introducción de RISS, podría generar problemas de inequidad en el acceso, si en el territorio no se mantiene el mismo grado de coordinación dentro y fuera de la red con los proveedores que conforman el sistema. En un contexto de competencia, podría mejorar la continuidad asistencial dentro de la red, si se garantiza el acceso ágil y oportuno de los afiliados a todos los niveles asistenciales. Pero la introducción de RISS también puede generar problemas en la equidad de acceso al sistema de salud, principalmente, debido a los incentivos para la selección de los pacientes y, a largo plazo, también suele producir una disminución en el acceso a la atención dentro de la red, por responder al incremento de costes limitando la cobertura de servicios (4) o introduciendo barreras a su utilización (44). En entornos competitivos, por tanto, requiere una fuerte regulación, que no llega a ser completamente efectiva en países desarrollados, y que hace dudar de su eficacia para países con escasa capacidad regulatoria por un funcionamiento débil del Estado, como podría ser el caso de países en vías de desarrollo (4) •

Agradecimientos. Este estudio ha sido parcialmente financiado por el Fondo de Investigación Sanitaria (FIS) PI042688 y el Consell Comarcal del Barcelonés Nord.

\section{REFERENCIAS}

1. Homedes N, Ugalde A. Why neoliberal health reforms have failed in Latin America. Health Policy 2005;71:83-96.

2.WHO. The World Health Report 2000. Health systems: improving performance. Geneva: WHO; 2000.

3. Mur-Veeman I, Van Raak A, Paulus A, Steenbergen M. Comparison and reflection. Integrated care in Europe.Maarsen: Elsevier; 2003. p. 169-90.

4. Vázquez ML, Vargas I, Farré J, Terraza R. Organizaciones sanitaria integradas. Una guía para el análisis. Rev Esp salud Pública 2005;79:633-43.

5. Vázquez ML, Vargas.I Redes integradas de servicios de salud: ¿ ¿Solución o problema? Ciencias de la Salud Bogotá (Colombia) 2006;4(1):5-9.

6. Shortell SM, Gillies RR, Anderson DA, Mitchell JB, Morgan KL. Creating organized delivery systems: the barriers and facilitators. Hosp Health Serv Adm 1993;38(4):447-66.

7. Hutchinson B, Hurley J, Reid R, Dorland J, Birch S, Giacomini M, et al. Capitation formulae for integrated health systems: a policy synthesis. Ottawa: Canadian Health Service Research Foundation; 1999.

8. Beck K. Competition under a regime of imperfect risk adjustment: the Swiss experience. Sozial-Präventivmedizin 1998;43:7-8. 
9. Jaramillo-Pérez I. El futuro de la salud en Colombia. Ley 100 de 1993 cinco años después. Bogotá: Frescol-Fes-FRB-Fundación Corona; 1999.

10. Van de Ven WP, Schut FT, Rutten FF. Forming and reforming the market for third-party purchasing of health care. Soc Sci Med 1994;39(10):1405-12.

11. Rico A, Moreno FJ. Arquitectura de las organizaciones sanitarias integradas en Europa. Integración asistencial: fundamentos, experiencias y vías de avance. Barcelona: Masson; 2006.

12. OPS. La salud en las Américas. Washington, DC.: OPS; 2002.

13. Jané E, Vázquez ML. El Sistema de Salud Español. Máster en Gestión de Centros y Servicios de Salud. Universitat de Barcelona, editor. 2002.

14. Mayolas E, Vargas I. La Sanitat en Catalunya: situació actual i perspectives de futur. Memòria Econòmica de Catalunya any 2002. Cambra Oficial de Comerç de Barcelona; 2003.

15. Agustí E, Casas E, Brosa F, Argimón JM. Aplicación de un sistema de pago basado en la población en Cataluña. In: Ibern P, editor. Integración asistencial: fundamentos, experiencias y vías de avance. Barcelona: Masson; 2006.

16. Conrad DA, Shortell SM. Integrated health systems: promise and performance. Front Health Serv Manage 1996;13(1):3-40.

17. Devers KJ, Shortell SM, Gillies RR, Anderson DA, Mitchell JB, Erickson KL. Implementing organized delivery systems: an integration scorecard. Health Care Manage Rev 1994;19(3):7-20.

18. Shortell SM, Gillies RR, Anderson DA. The new world of managed care: creating organized delivery systems. Health Aff (Millwood ) 1994;13(5):46-64.

19. Coderch J. Serveis de Salut Integrats Baix Empordà (SSIBE): elementos clave en la evolución del hospital a la organización sanitaria integrada. En: Ibern P, editor. Integración asistencial:fundamentos, experiencias y vías de avance.Barcelona: Masson; 2006. p. 139-86.

20. Vargas I, Vázquez ML. Barreras y facilitadores de la coordinación asistencial en dos organizaciones sanitarias integradas. Gac Sanit 2007;21(2).

21. Vázquez ML, Vargas I. Organizaciones sanitarias integradas. Un estudio de casos. Barcelona: Consorci Hospitalari de Catalunya CHC; 2007.

22. Patton Q. Qualitative evaluation and research methods. London: Sage; 1990.

23. Vargas I, Vázquez ML, Farré J. Guía para el estudio de Organizaciones Sanitarias Integradas. 2004. Consorci Hospitalari de Catalunya. CHC.

24. Vázquez ML, Vargas I, Terraza R, Pizarro V. Marco conceptual para el estudio de las Organizaciones Sanitarias Integradas. En: Vázquez ML, Vargas I (Ed.). Organizaciones Sanitarias Integradas. Un estudio de casos. Barcelona: Consorci Hospitalari de Catalunya CHC; 2007. p. 7-31.

25. Badalona Serveis Assistencials. Memoria 2003. Badalona: Badalona Serveis Assistencials; 2003. 
26. Institut d'Estadistica de Catalunya. [Internet]. Disponible en: http://www.idescat.net/ cat/poblacio/poblestructura.html. Consultado Mayo de 2005.

27. Institut Català de la Salut. Centres i Serveis. [Internet]. Disponible en: http:// www.gencat.net/ics/usuaris/centres_serveis.htm . Consultado Mayo de 2005.

28. Institut Municipal de Serveis Personals (IMSP). Serveis de salut mental d'adults. Ajuntament de Badalona [Internet]. Disponible en: http://www.badalona.cat .Consultado Mayo de 2005

29. Instituto de Asistencia Sanitaria. Baix Empordà. IAS [Internet]. Disponible en: www.ias.scs.es. Consultado Mayo de 2004

30. Servicio de atención primaria Gerona - Empordà - Sant Feliu de Guíxols. ICS. [Internet]. Disponible en: www.gencat.net/ics/usuaris/centres_servicios.htm. Consultado Junio de 2004

31. Serveis de Salut Integrats Baix Empordá. Sistemes de informació per a la integració de serveis. Palamós: Serveis de Salut Integrats Baix Empordà; 2002.

32. Castaño RA. Integración vertical entre empresas promotoras de salud e instituciones prestadoras de servicios de salud. Gerencias y políticas de salud 2004;3(006):3551.

33. Pané O, Segú JL, Via JM. Descripción y valoración de alternativas de cambio en los sistemas sanitarios basadas en la potenciación de atención primaria en salud. 1997. Consoci Hospitalary de Catalunya CHC CiG.

34. Shortell SM, Gillies RR, Anderson DA, Erikson D, Mitchell J. Remaking health care in America. Building organized delivery systems. 2nd ed. San Francisco: The Jossey-Bass health care series; 2000.

35. Vargas I. La utilización del mecanismo de asignación per cápita: la experiencia de Cataluña. Cuadernos de gestión para el profesional de atención primaria 2002;8(4):167-78.

36. Terraza R. Factors que afavoreixen i obstaculitzen la implantació d'un sistema de compra de base poblacional a Catalunya [tesina de doctorado] Barcelona: Departamento de pediatría, obstetricia y ginecología y de medicina preventiva, Universidad Autónoma de Barcelona; 2007.

37. Aday LA, Andersen RM. A framework for the study of access to medical care. Health Service Research 1974;9(3):208-20.

38. Pointer DD, Ewell CM. Really governing: what type of work should boards be doing? Hosp Health Serv Adm 1995;40(3):315-31.

39. Alexander JA, Zuckerma HS, Pointer DD. The challenges off governing integrateed health care systems. Health Care Manage Rev 1995;20 (4):69-81.

40. Vargas I, Vázquez ML. Análisis comparativo. In: Vázquez ML, Vargas I, editors. Organizaciones sanitarias integradas. Un estudio de casos. Barcelona: Consorci Hospitalari de Catalunya CHC; 2007. 
41. Shortell SM, Gillies R.R., Anderson DA, Morgan KL, Mitchell J.B. Remaking health care in America. $1^{\text {a }}$ ed. San Francisco: The Jossey-Bass health care series; 1996.

42. Gillies RR, Shortell SM, Young GJ. Best practices in managing organized delivery systems. Hosp Health Serv Adm 1997;42(3):299-321.

43. Henao D, Vázquez ML, Vargas I, Coderch J, Colomès Ll, Llopart JR, et al. Coordinación asistencial en las organizaciones sanitarias integradas según directivos y profesionales. Gac Sanit 2007;21 (Supl 2):63.

44. Vargas I, Vázquez ML, Mogollón A. El acceso a la atención a la salud en modelos de competencia regulada: Un estudio de caso en Colombia. Gac Sanit 2007; 21 (Espec Congr):146. 